\title{
La historiografía ecuatoriana contemporánea
}

\author{
Jorge Núñez Sánchez \\ Academia de Historia y Geografía \\ Casa de la Cultura Ecuatoriana
}

A comienzos de la década de los setenta surgió en Ecuador un movimiento de renovación historiográfica conocido como "Nueva historia". Empeñado en construir su propia visión del pasado ecuatoriano a partir de otras perspectivas de análisis, este movimiento produjo desde entonces una variada bibliografía, que refleja las ideas, inquietudes y limitaciones de una generación de historiadores ecuatorianos, en general críticos de la vieja escuela y del anquilosado academicismo oficial. Una evaluación de los resultados alcanzados por este movimiento intelectual muestra algunos significativos logros. Nunca antes se había investigado tanto ni incursionado en tantos temas y campos de estudio. Igualmente, no se había publicado tanto como lo ha hecho este movimiento, cuya producción incluye una "Nueva historia del Ecuador", ambiciosa publicación en quince tomos. Complementando a la nueva historiografía ecuatoriana está la labor de los historiadores ecuatorianistas, que han aportado estudios de gran utilidad para la comprensión del pasado ecuatoriano.

\section{La búsqueda de una renovación historiográfica}

En 1976, durante un simposio organizado por la Universidad Central del Ecuador, presentamos un trabajo titulado "Problemas de la historiografía y la enseñanza de la historia del Ecuador”, en el que señalábamos como principales características de la historiografía vigente en el Ecuador las siguientes:

1. "El carácter adjetivo y superficial... que pone énfasis en la recopilación de datos y mención cronológica de hechos, así como en la descripción de los aspectos exteriores de los mismos, antes que en el análisis crítico de su carácter, contenido y trascendencia".

2. "La relativa pobreza temática, que saltaba a la vista con sólo mirar la bibliografía existente, y una aberrante tendencia... a volver repetidamente sobre los temas de siempre, casi siempre con el único objetivo de glosar las opiniones ya vertidas o relievar algún aspecto secundario...".

3. "El empirismo metodológico y la generalizada falta de conocimientos metodológicos y técnicos por parte de los historiadores nacionales...".

4. "La persistencia de grandes nudos historiográficos", que había determinado que la mayor parte de la bibliografía existente se concentrara 
"en el análisis de unos pocos períodos, que se privilegiaban sobre otros, y que básicamente eran: la Conquista, la Independencia, el Garcianismo y la Revolución Liberal”.

5. Una "estrecha vinculación con el Estado y las clases dominantes en él representadas, ... (quienes) habían utilizado a la Historia como mecanismo de justificación de su poder". Agregábamos que parte de ese "ejercicio del poder de clase en el campo de la Historia había sido la creación de un aparato institucional destinado a la elaboración historiográfica”.

6. Un desorbitado culto a los héroes.

En cuanto a las características de la enseñanza de la Historia en el país, precisábamos como las más significativas las siguientes: pobre nivel de los programas de enseñanza; improvisación docente; privilegio de la "historia ideológica" frente a la "historia científica"; carácter memorístico de la enseñanza; falta de relación del cuerpo docente con la investigación; e inexistencia de sistemas de reciclaje y capacitación profesional para los maestros.

Ante tan poco estimulante panorama, se volvía imprescindible una profunda y generalizada renovación historiográfica, que tendiera a la búsqueda de un creciente nivel científico en los estudios históricos y estimulara la profesionalización de la labor investigadora. A su vez, todo ello debería reflejarse en la producción de una nueva bibliografía y en un enriquecimiento general de los conocimiento sobre el pasado nacional, toda vez que la historia, a diferencia de otras ciencias, rebasa normalmente el ámbito de los círculos especializados y concita el interés de toda la ciudadanía.

El desarrollo de una moderna historiografía ecuatoriana, que las gentes de mi generación asumimos originalmente con mucho voluntarismo, habría de revelársenos en la práctica como una tarea a largo plazo, tanto más cuanto que partíamos de una muy endeble base institucional, contábamos con pocos recursos humanos, técnicos y materiales, y debíamos superar la inercia y resistencia de la vieja historiografía. Entre los puntos a nuestro favor contábamos con el entusiasmo de la juventud y el apoyo de unos poquísimos pero valiosos historiadores de la vieja escuela.

La buscada renovación de los estudios históricos tuvo un notable antecedente en 1971, cuando el destacado científico social Agustín Cueva, recientemente fallecido, publicó su libro El proceso de dominación política en Ecuador, que ganara una mención de honor del Concurso de Ensayo Casa de las Américas. A ello siguió la aparición de otras dos obras matinales 
de las ciencias sociales ecuatorianas: Ecuador: pasado y presente (1975), del Instituto de Investigaciones Económicas de la Universidad Central, y Ecuador: subdesarrollo y dependencia, de Fernando Velasco Abad.

Desde luego, no fue casual que el alumbramiento de la moderna historiografía ecuatoriana haya correspondido a los sociólogos y no a los historiadores. Y no lo fue por varias razones: una, el momento histórico que vivía América Latina, tras el formidable remezón de la Revolución Cubana, que generó en sus vanguardias intelectuales una notoria preferencia por la sociología, la politología o la economía, antes que por la historia; otra, el carácter empírico y extremadamente conservador que por entonces tenía en el Ecuador el mundo de los historiadores, integrado por gentes identificadas con el más conspicuo tradicionalismo social y político, y que además habían sido incapaces de instituir al menos una escuela de estudios históricos.

Pero la intrínseca importancia que revestía el análisis histórico para un mejor conocimiento de nuestra sociedad nacional determinó que, de entre la misma vanguardia intelectual de izquierda, surgiera en Ecuador una nueva corriente historiográfica, que buscó revisar las viejas concepciones e incorporar nuevos temas y perspectivas de estudio.

Hecho importante para la historiografía ecuatoriana fue, por aquellos años, la creación del Instituto de Investigaciones Regionales de la Universidad de Cuenca (IIRDUC), posteriormente denominado Instituto de Investigaciones Sociales (IDIS), que dió un impulso a la investigación histórica, gracias a la presencia de algunos investigadores argentinos y chilenos llegados con los vientos del exilio. ${ }^{1}$ Así, a partir de 1978 se institucionalizó en Cuenca el "Encuentro de Historia y Realidad Económica y Social del Ecuador", que tendría nuevas reuniones en los años 1980, 1986, 1989 y 1991, convirtiéndose en un importante espacio de análisis y coordinación del trabajo de los científicos sociales del país. Y es que, salvo excepciones, durante los años setenta no se produjo en Ecuador una clara diferenciación entre la investigación histórica y el trabajo sociológico, tanto por la carencia de una verdadera tradición de profesionalismo entre los historiadores, cuanto por el interés que había, de parte de los partidos de izquierda, en utilizar la "interpretación histórica" como elemento de justificación y consagración de sus contrapuestas corrientes políticas.

1 A riesgo de olvidar algunos nombres, mencionamos los de Horacio Cerutti Guldberg, Silvia Palomeque, María Cristina Cárdenas y Gerardo Aceituno. 
Carente de escuelas universitarias de Historia, afectado por una pobreza de publicaciones especializadas y una general carencia de rigor en los estudios e investigaciones, el mundo de los historiadores estaba en total crisis. El único gran historiador superviviente de las anteriores generaciones era, paradójicamente, un notable autodidacta y afamado literato, cuya obra intelectual se había desarrollado al margen de las empobrecidas Academias de la Historia y de la Lengua y, en esencia, a contrapelo de éstas: don Alfredo Pareja Diezcanseco.

Esta realidad preexistente determinó que la irrupción del "sociologismo histórico" - que aportaba nuevas inquietudes y herramientas metodológicas al quehacer historiográfico, pero que por otro lado despreciaba la investigación de archivo y privilegiaba un interminable debate acerca de categorías y conceptos teóricos - no tuviera contrapeso ninguno y que los nuevos estudiosos de la historia ecuatoriana no pudieran contar con una adecuada formación u orientación profesional, ni debieran enfrentar una exigente emulación generacional. Sería sólo más tarde, bajo los impulsos de profesionalización de la naciente nueva escuela historiográfica, cuando los estudios históricos lograrían adquirir una creciente autonomía teórico-metodológica y liberarse progresivamente del sociologismo, aunque conservando en buena medida el bagaje instrumental aportado por éste.

Uno de los primeros pasos hacia la profesionalización de los historiadores fue la creación, en 1986, de la Asociación de Historiadores del Ecuador (ADHIEC), filial ecuatoriana de la Asociación de Historiadores Latinoamericanos y del Caribe (ADHILAC). Dadas las circunstancias expuestas, la nueva asociación se integró con científicos sociales de las más diversas especialidades, lo que en el futuro se mostraría como una traba para su desarrollo.

A su vez, en Quito se abría un nuevo espacio para la reflexión histórica con la creación del Encuentro Nacional de Historia (1980), evento que en el futuro tendría una convocatoria anual.

También devendría importante estímulo al desarrollo de la historiografía ecuatoriana la radicación en Ecuador de la Secretaría Ejecutiva de la ADHILAC. Ello estimuló la creación de la ADHIEC y, a través de varias actividades de promoción, contribuyó a estimular la investigación histórica y la publicación de sus resultados, y a difundir en el país la historiografía latinoamericana o "latinoamericanista" más reciente. El octavo Encuentro de Historia Nacional (1991) fue paralelamente el Primer Encuentro de 
Historia Andina y contó con la participación de historiadores latinoamericanos y de latinoamericanistas europeos.

\section{Influencias y orientaciones}

En la circunstancia descrita, la nueva producción historiográfica ecuatoriana obedeció a diversas influencias teóricas y orientaciones metodológicas. Una influencia notoria fue la del marxismo, precisamente porque aportaba una visión estructural de la sociedad nacional y mundial, capaz de dar respuestas a una ya endémica situación continental de atraso y dependencia, que la Revolución Cubana se había encargado de revelar en toda su angustiosa magnitud. Pero, en general, el "marxismo vulgar" en uso estuvo cargado de un aberrante reduccionismo, que privilegiaba a las clases y sus luchas como únicas fuerzas motrices de la historia, e ignoraba deliberadamente todo el movimiento social producido al margen de aquéllas, tal como el de las etnias y nacionalidades minoritarias, que en países pluriétnicos y pluriculturales, como los latinoamericanos, tienen una notable presencia histórica.

Influencias significativas han sido también las de la escuela francesa de los Annales, la de la historiografía anglosajona y la de la escuela latinoamericana de historia de las ideas, orientada por estudiosos como Leopoldo Zea, Arturo Andrés Roig y Rodolfo Agoglia, estos últimos residentes en Ecuador durante su exilio político. ${ }^{2}$ Todas esas influencias se concretaron particularmente en la orientación de los grupos de estudio, centros de investigación y líneas editoriales constituidos en el país desde fines de los setenta.

\section{Viejas y nuevas especialidades}

La Historia Política.-Tradicionalmente vigorosa en el país, alcanzó en el período algunos logros notables, que pueden resumirse en tres: la superación de la cronología tradicional, el abandono de la "historiografía partidista" y un enriquecimiento temático que buscaba dejar atrás los

2 Durante su estancia en el país, Roig fundó y dirigió el CELA de la Pontificia Universidad Católica de Quito (PUCE) y desarrolló o publicó algunos importantes estudios sobre el pensamiento latinoamericano y ecuatoriano. 
"grandes nudos historiográficos". En ese marco, una primera clarinada vino con la publicación de dos libros ya "clásicos" en este campo: El poder político en Ecuador, de Osvaldo Hurtado Larrea, un científico social democristiano, que en el futuro sería presidente de la República, y Lucha política y origen de los partidos en el Ecuador, de Enrique Ayala Mora. A su vez, desde la sociología llegó el mismo año un interesante aporte con la publicación del libro de Augusto Varas y Fernando Bustamante Fuerzas armadas y política en el Ecuador.

A partir de entonces se desarrolló vigorosamente esta especialidad, ofreciendo logros de diversa magnitud. Entre los autores destacamos los nombres de Elías Muñoz Vicuña, Manuel Medina Castro y Julio Estrada Icaza, pertenecientes a una generación anterior, y los de Patricio Martínez Jaime, Juan Paz y Miño, Jorge Núñez, Francisco Dávila Aldas, Pilar Ponce Leiva, Silvia Vega Ugalde, Wellington Paredes, Alexei Páez. Últimamente, la pareja intelectual y afectiva formada por Erika Silva y Rafael Quintero han lanzado su ambiciosa obra Ecuador: una nación en ciernes, que busca explicar la historia ecuatoriana desde la perspectiva de la cuestión nacional.

Nuevos temas y nuevas perspectivas de análisis han enriquecido el tratamiento de la historia política contemporánea. Uno de ellos ha sido el del populismo, que por su misma notoria gravitación en la vida nacional mantiene una permanente novedad en el ámbito intelectual. Hasta hace poco, el tratamiento de este tema ha estado casi exclusivamente en manos de sociólogos: lo inició Agustín Cueva —que, también en esto, marcó una huella pionera - con un breve pero fundamental estudio sobre el velasquismo; posteriormente saldrían a la luz los trabajos de Rafael Quintero, Pablo Cuvi y otros. En los últimos años, el tema del populismo ha sido rescatado para la historia política por Juan Paz y Miño, mientras que Juan Maiguashca lo ha analizado desde la perspectiva de la diferenciación económica regional y sus consecuencias sociales.

Otros temas que han atraído la atención de los historiadores han sido la historia de los partidos políticos (Ayala, Núñez), el período constitutivo del Estado ecuatoriano (Núñez, Vega Ugalde), y las revoluciones y revueltas populares (Muñoz Vicuña, Estrada Icaza, Martínez, Vega Ugalde). El tema del dictador, que tanto ha marcado la cultura latinoamericana de los últimos dos siglos, sigue interesando a la literatura, a la sociología y, aunque en menor medida, a la historia, desde la cual han aflorado últimamente un excelente estudio de Pilar Ponce Leiva sobre Gabriel García Moreno, y otros de Gonzalo Ortiz Crespo sobre el "Febrescorderato", estos últimos 
a medio camino entre la crónica y la historia inmediata. En líneas generales, el tema del Estado y su historicidad se ha mostrado particularmente atractivo para los sociólogos, y en especial para Osvaldo Hurtado, Patricio Moncayo, Rafael Quintero y Erika Silva, Alejandro Moreano, José María Egas y Daniel Granda.

No podemos cerrar este capítulo sin mencionar ciertos importantes estudios históricos sobre las fuerzas armadas publicados en el período que nos ocupa: Las Fuerzas Armadas: de la Revolución Alfarista al Movimiento Juliano, del actual general Paco Moncayo Gallegos, y Las Fuerzas Armadas Ecuatorianas: paz y desarrollo, del coronel Alberto Molina Flores, que analizan "desde adentro" y con una perspectiva socio-política la evolución institucional de los cuerpos militares, sus acciones políticas y la mentalidad antioligárquica que las sustenta.

La Historia de lo Social.-Una de las nuevas especialidades desarrolladas en el período ha sido la historia de lo social, que se iniciara en los años sesenta con la obra pionera de dos etnohistoriadores, los esposos Piedad y Alfredo Costales, del historiador autodidacta Oswaldo Albornoz Peralta y del sociólogo Jaime Galarza Zavala. Posteriormente, esta especialidad se desarrolló en el país bajo el impulso teórico del marxismo, de la etnología y de la escuela inglesa de historia social. Entre los autores más nuevos podemos mencionar a Andrés Guerrero, Manuel Chiriboga, Patricio Martínez, Jorge Trujillo, Hernán Ibarra, Leonardo Espinoza, Claudio Cordero, Jorge Núñez, Patricio Icaza, Juan Paz y Miño, Lucas Achig y Milton Luna, en cuya labor intelectual se puede detectar una combinación de influencias teóricas y una búsqueda de nuevas rutas metodológicas hacia la aprehensión de los rasgos esenciales de la vida colectiva. En los últimos años, han incursionado en este ámbito algunos jóvenes historiadores, como Patricia de la Torre, Rosario Coronel Feijóo, Silvia Benítez, Guillermo Bustos y Rocío Rueda. Tamara Estupiñán, pese a su juventud, ha producido un importante estudio de historia de la familia, que marca un hito metodológico en la especialidad.

En el ámbito temático, el sistema de dominación y las clases dominantes han merecido un particular interés de los nuevos historiadores. También ha sido un tema de notorio atractivo la historia de la oligarquía y ello ha estado motivado, obviamente, por la fuerza y persistencia del poder oligárquico en el país. Entre los varios trabajos historiográficos que el tema ha suscitado, los hay sobre la oligarquía ecuatoriana en general (Núñez), 
sobre las oligarquías regionales en particular (Rafael Guerrero, Trujillo), sobre la política y el discurso oligárquico (Martínez), y sobre las relaciones sociales al interior del sistema de la hacienda (Chiriboga, A. Guerrero, Patricia De la Torre). Paralelamente, se han desarrollado unos pocos estudios sobre la clase burguesa (R. Guerrero, Luna).

La historia del movimiento obrero mereció en el período reseñado una especial atención de los historiadores, llegando a convertirse en uno de los nuevos "temas centrales" de la historiografía ecuatoriana. Sin otro antecedente que los estudios del dirigente comunista Pedro Saad sobre la Confederación de Trabajadores del Ecuador, algunos historiadores de la nueva generación se empeñaron en analizar la historia de las organizaciones laborales en el siglo XX. Así se desarrollaron y publicaron importantes obras generales, como las de Patricio Icaza, Jaime Durán Barba y Oswaldo Albornoz Peralta, y ensayos sobre temas especializados, como los de Alexei Páez, Jorge León y Jorge Oviedo. También se constituyeron equipos de estudio, tales como el que formó el IDIS, bajo la dirección de Leonardo Espinoza, y con participación de Juan Paz y Miño, Manuel Medina Castro, Lucas Achig y Jorge Núñez.

Este variado esfuerzo ha permitido ampliar sustancialmente el conocimiento preexistente sobre el movimiento obrero ecuatoriano, sus organizaciones y luchas. Empero, ha adolecido en general de las distorsiones propias de una visión política interesada, dado que la mayoría de historiadores del movimiento obrero se halla vinculada a las diversas organizaciones sindicales y se ha empeñado en destacar las acciones o planteamientos de una u otra; por el mismo motivo, se han sobredimensionado algunos fenómenos o dejado de lado temas o períodos de estudio carentes de directo interés político. Una notable excepción ha constituido el trabajo de Patricio Martínez Jaime sobre la insurrección popular de noviembre de 1922, por la profesionalidad del estudio realizado y por la nueva perspectiva que abrió al incorporar el análisis del discurso político.

En general, tanto por los planteamientos como por los resultados, podemos concluir que el desarrollo de la historia de lo social recreó el escenario visible del pasado ecuatoriano. Al incluir en el panorama historiográfico nuevos temas de interés, categorías de análisis y formulaciones metodológicas, e inclusive nuevas técnicas de investigación, consiguió que éste dejara de estar poblado únicamente por conquistadores, santos, héroes, caudillos, dictadores y líderes políticos, para pasar a enriquecerse con la presencia de los actores sociales: clases, etnias, categorías socio-profesio- 
nales, "masas populares" y sectores sociales subordinados o marginados de la historia (indios, cholos, trabajadores, mujeres). Gracias a la irrupción de estas nuevas perspectivas de análisis, la historia dejó de ser un "escenario político" para convertirse en un "escenario social", donde las fuerzas motoras del movimiento histórico ya no serían las ideas de los líderes, las confrontaciones de las individualidades palaciegas o los conflictos partidarios, sino los intereses, anhelos o pasiones colectivas, enfrentados en luchas clasistas, interclasistas, interétnicas o regionalistas.

Consideramos necesario referirnos al gran impulso que ha cobrado en la última década una de las subespecialidades de la historia de lo social: la Genealogía. Si la búsqueda de identidad es una tendencia natural de todo grupo social, la identificación de sus ancestros es una preocupación que subyace en cada espíritu humano. Así, todo aquel que se aproxima al estudio de las genealogías - especialista o no- siente vivir en un mundo del cual es partícipe, sujeto y objeto a la vez. Eso explica el hecho de que, siguiendo la huella del gran genealogista guayaquileño Pedro Robles Chambers y bajo el estímulo de algunos apasionados cultores actuales del genealogismo - tales como Fernando Jurado Noboa y Juan Freile Granizo- se hayan constituido dos vigorosas asociaciones de estudios genealógicos, formadas por unos pocos genealogistas profesionales y un extenso número de colaboradores e informantes: la Sociedad Amigos de la Genealogía, dirigida por Jurado, y el Centro Nacional de Investigaciones Genealógicas y Antropológicas, dirigido por Freile. Además de organizar encuentros periódicos de sus miembros, estas entidades efectúan una activa política de publicaciones.

Un importante aporte hecho a la historiografía por el movimiento genealogista ha sido el estudio de los orígenes indígenas o negros de las familias ecuatorianas, lo cual ha servido para demostrar, en última instancia, el carácter temprana y profundamente mestizo de nuestra sociedad.

Emparentada con la genealogía y la demografía, pero con ribetes propios, se ha ido desarrollando la obra de Jorge Moreno Egas, un serio investigador de la historia socio-urbana y los estamentos sociales. Por su lado, la historia de las mentalidades tiene cultores de prestigio en el mismo Moreno Egas, en Andrés Guerrero y en Ernesto Salazar.

Por fin, es necesario referirse a dos temáticas adicionales: la historia de la mujer y la historia de la inmigración. En cuanto a la primera, preciso es señalar que en las últimas décadas ha tenido apenas un tímido despegue, en buena medida gracias a la labor aislada de dos historiadoras: Cristiana 
Borchart, en Quito, y Jenny Estrada, en Guayaquil, a las que se ha unido últimamente Jenny Londoño, autora de un importante estudio sobre las mujeres en el ocaso colonial. Salta a la vista que el escaso desarrollo de esta especialidad en el Ecuador se debe principalmente a la falta de interés de las propias organizaciones feministas por el asunto.

Respecto de la historia de la inmigración, las únicas personas que han incursionado profesionalmente en ella son, hasta el momento, Jenny Estrada y Armando Otatti, aunque, como aporte testimonial, no deja de ser útil el libro de Henry Raad sobre la inmigración árabe.

La Etnohistoria.-Según la cronología del desarrollo historiográfico, podemos decir que los temas clasistas tuvieron un interés predominante en las décadas de los sesenta, setenta y comienzos de los ochenta, pero que en el último decenio el interés preponderante se centró en los asuntos étnicos. De paso, esto último ha significado una suerte de redescubrimiento del país, al mismo tiempo que la emergencia de un nuevo enfoque epistemológico, que nos ha llevado desde las preocupaciones clásicas de la historiografía occidental (el movimiento obrero, las luchas campesinas, etc.) a temáticas más propias de nuestra realidad social, como las de los indios, los negros o los mestizos. Obviamente, ese nuevo enfoque ha sido estimulado por las urgencias de la realidad, puesto que, aproximadamente desde mediados de la década pasada, los indios irrumpieron como nuevos sujetos históricos en el escenario de la vida nacional y obligaron a un replanteamiento de todo el pensamiento social, tanto académico como político.

En este período y circunstancia, la etnohistoria ha alcanzado un notable salto cualitativo y cuantitativo, al calor de la emergencia política de las nacionalidades indígenas, que en estos años han ido convirtiéndose en uno de los más activos e influyentes movimientos sociales del Ecuador contemporáneo. También ha pesado en ello el desarrollo de la etnología andina, que ha devenido una de las más sugerentes utopías político-intelectuales contemporáneas. Tras la amplia trocha abierta por Segundo Moreno Yánez y Hugo Burgos, han seguido esta ruta nuevos investigadores, provenientes tanto de la historia como de la sociología, la antropología y la medicina. Entre ellos están la etnóloga Ileana Almeida, promotora del desarrollo de las culturas indias, el etnohistoriador Galo Ramón, el sociólogo Hernán Ibarra, y los antropólogos Jorge Trujillo — creativo cultor de la investigación-acción, Blanca Muratorio, Juan Botasso, Carlos Coba y José 
Sánchez Parga, así como los jóvenes historiadores Cristóbal Landázuri y Xavier Andrade.

Fenómeno trascendental ha sido, en los últimos años, la aparición de un vigoroso movimiento intelectual indígena, algunos de cuyos miembros han publicado trabajos de etnohistoria o reflexión etnohistórica, como parte de la lucha de reivindicación nacional de su pueblo: Nina Pacari, Ariruma Koowi, José Quimbo y Luis Maldonado.

La Historia Económica.-Al comenzar el período de nuestro análisis, ésta era una absoluta novedad en el Ecuador, sin otros antecedentes que los estudios de Víctor Emilio Estrada y la posterior Historia monetaria y cambiaria del Ecuador, de Luis Alberto Carbo. Sin embargo, ha tenido en las últimas dos décadas algunos cultores, como Gonzalo Ortiz Crespo, Manuel Chiriboga, Andrés Guerrero, Christiana Borchart, Manuel Miño Grijalva, Juan Maiguashca, Hugo Arias, Alberto Acosta, Willington Paredes y Nicanor Jácome. Particularmente importante ha sido la labor de Carlos Marchán Romero, permanente animador del desarrollo de esta especialidad, fundador y director de la Revista Ecuatoriana de Historia Económica, excelente publicación del Banco Central del Ecuador, que hoy se halla lamentablemente descontinuada. A la vez, en Guayaquil ha venido actuando un grupo de historiadores económicos de formación liberal clásica, entre los que destacan Julio Estrada Icaza $(\dagger)$, director del Archivo Histórico del Guayas, y Guillermo Arosemena. De entre los historiadores más jóvenes que han incursionado en la historia económica deben mencionarse los nombres de Rosemarie Terán y Guadalupe Soasti. Un trabajo solitario e individual ha sido el de Carlos Ortuño, autor de una interesante Historia numismática del Ecuador.

Una evaluación desapasionada de los logros alcanzados en nuestro país en el campo de la historia económica demuestra que son todavía escasos, aunque algunos han alcanzado un nivel ciertamente respetable. De otra parte, están todavía por estudiarse algunos fenómenos trascendentales de nuestra historia económica, tales como la mutua articulación de las economías regionales, sus diversas formas y ritmos de vinculación al mercado internacional, los ciclos de auge y crisis en las pequeñas economías regionales, los circuitos económicos fronterizos, entre otros muchos. Y mientras esos estudios no se realicen, todavía estaremos incapacitados para entender plenamente otros fenómenos históricos trascendentales, como el federalismo y las guerras civiles, el regionalismo, la migración campo-ciudad o el contrabando. 
En síntesis, y en una apreciación general, podemos afirmar que la historia económica no ha logrado todavía "cuajar" una vigorosa corriente ni ha creado escuela en el país, circunstancia que obviamente afecta al desarrollo global de los estudios históricos, pues aún no hemos logrado acumular una reveladora suma de conocimientos objetivos sobre el pasado de la economía ecuatoriana.

La Historia Demográfica.-Si la historia económica ecuatoriana es pobre, la historia demográfica prácticamente no existe, salvo algunos aislados ensayos sobre temas muy particulares, los cuales, en todo caso, no se proponen explicar los grandes procesos o fenómenos demográficos de nuestro pasado. En medio de tal desierto se alzan solitarios los breves ensayos de Jorge Moreno Egas o Silvia Benítez, que, sin duda, exigen un esfuerzo continuado de sus autores en esta línea de investigación.

La Historia de la Cultura.-Dentro del general proceso de renovación historiográfica del período, una línea muy sostenida de trabajo ha sido la de la historia de la cultura y, dentro de ella, la historia de las ideas y las mentalidades.

Los pioneros en este campo surgieron en la década de los setenta, aunque sería mejor decir que "insurgieron" entonces, pues su obra nació como un cuestionamiento a las estructuras de dominación y a su aparato ideológico. El pionero principal fue, una vez más, el brillante Agustín Cueva (†), cuyo libro Entre la ira y la esperanza marcó un corte decisivo en el estudio de la historia de la cultura ecuatoriana. Contemporáneos suyos fueron los otros pioneros en la especialidad: Fernando Tinajero, Ernesto Albán Gómez y Vladimiro Rivas.

A partir de la década de los ochenta, cobró fuerza en el país la historia de las ideas, notablemente influida por Leopoldo Zea y, sobre todo, por Arturo Andrés Roig. Durante sus varios años de residencia en el país, Roig llegaría a formar, junto con Rodolfo Agoglia, una verdadera escuela de pensamiento, principalmente a través de sus cátedras en la PUCE y en la Universidad Central del Ecuador. En la actualidad, sus cultores se hallan nucleados básicamente alrededor de centros investigadores de las universidades nacionales. El más importante de ellos se halla en la PUCE y fue propiciado originalmente por Hernán Malo González S.J., brillante pensador católico (†), en la época en que ejerció el rectorado de esta Universidad. Está integrado por un grupo de notables intelectuales: Carlos Paladines, 
animador y director de la Revista Ecuatoriana de Historia de las Ideas, ${ }^{3}$ Samuel Guerra Bravo, Carlos Landázuri Camacho, Jorge Villalba S.J., Nancy Ochoa Antich, Susana Cordero de Espinoza y Ernesto Salazar, este último de la generación más joven.

Un equipo importante es también el que fundaran en la Universidad de Cuenca Alfonso Carrasco, Horacio Cerutti Guldberg, María Cristina Cárdenas y Claudio Cordero, integrado luego por Jorge Dávila Vásquez, destacado escritor e historiador de la cultura, María Augusta Vintimilla, Adrián Carrasco, María Elena Albán y otros. También en Cuenca, pero en la joven Universidad del Azuay, existe un equipo similar, del que forman parte Claudio Malo González, Juan Cordero Iñiguez y Marco Tello Espinoza. Por fin, en la Universidad Central laboran, aunque sin formar equipo, algunos intelectuales que trabajan en la temática de la historia de la cultura, entre ellos Alejandro Moreano y Fernando Tinajero.

En esa misma línea se inscriben los esporádicos esfuerzos del Instituto de Investigaciones de la Cultura Ecuatoriana, que nuclea a un grupo de prestigiosos intelectuales quiteños: Francisco Proaño Arandi, José Ron, Iván Carvajal, Humberto Vinueza, Milton Benítez y Luis Corral.

Mención especial merecen en la historia de la cultura ecuatoriana la labor investigadora y analítica de Lenin Oña, afamado crítico e historiador del arte; de Hernán Rodríguez Castelo, multifacético historiador de la cultura ecuatoriana; de Alexandra Kennedy Troya, prestigiosa investigadora de la historia del arte y la artesanía; de Alfonso Ortiz Crespo, historiador del arte y la arquitectura; de Juan Valdano, estudioso de las generaciones culturales, y de Ximena Escudero de Terán, estudiosa del arte colonial quiteño.

La Historia Urbana y Regional.-Especialidades que han adquirido creciente importancia durante el período son la historia urbana y la historia regional, cuya florescencia contemporánea responde tanto a intereses concretos de la vida social cuanto a inquietudes estrictamente intelectuales. Su primer impulso vino desde el Archivo Histórico del Guayas, con los estudios de Abel Romeo Castillo y de Julio Estrada Icaza, prestigiosos historiadores de la anterior generación. Luego retomó esa línea de trabajo el IDIS de Cuenca, gracias al entusiasmo intelectual de Silvia Palomeque, Leonardo Espinoza, Lucas Achig, Juan Chacón, Julio Carpio y Paciente

3 La revista es coeditada por el CELA de la PUCE y la CCE; su director es Carlos Paladines Escudero.

Tomo LIII, 1, 1996

289 
Vásquez, autores de importantes estudios sobre la historia de la región austral y de su capital histórica, Cuenca.

También en la década de los setenta comenzó su labor el Instituto Otavaleño de Antropología (IOA), cuya labor estimularía los estudios de historia regional, etnohistoria y arqueología. De otro lado, el despegue de esta especialidad fue estimulado por Juan Maiguashca, historiador ecuatoriano residente en Canadá, a través del Centro de Estudios Latinoamericanos de la Universidad de York.

En la actualidad, los más importantes promotores de la historia urbana son el centro de investigaciones CIUDAD (Quito), donde laboran en el tema varios investigadores (Fernando Carrión, Eduardo Kingman, Ana María Goetschel y Patricio Velarde), la prestigiosa revista de arquitectura y urbanismo Trama, editada en Quito por Rolando Moya y Evelia Peralta, y la Corporación de Estudios Regionales Guayaquil (CER-G), dirigida por Gaitán Villavicencio, a la que se hallan vinculados Milton Rojas, Pablo Lee, Letty Chang y Graciela de Vélez. Producto de una labor aislada, pero no por ello menos meritoria, es la obra de Carlos Maldonado P., autor de varios ensayos sobre historia de la arquitectura.

En el mismo ámbito cabe situar la labor de Rubén Moreira y Alfonso Ortiz Crespo, historiadores de la arquitectura y el urbanismo, de Patricio Martínez Jaime, dirigente de la ADHIEC y autor de un importante estudio sobre la evolución del sector informal urbano, y de Alfredo Lozano Castro, autor de sugerentes estudios de etno-urbanismo. Otros investigadores que actúan en este campo son Inés del Pino, Jorge Benavides Solís, Lucas Achig, Martha Moscoso, Cecilia Mantilla y Sonia Fernández.

En cuanto a la historia regional, que otrora impulsaran intelectuales de la talla de Octavio Cordero Palacios, Pío Jaramillo Alvarado, Modesto Chávez Franco y Pedro Robles Chambers, y que hasta hace poco tuviera cultores tan entusiastas como Julio Estrada Icaza y Abel Romeo Castillo (Guayas), Hernán Gallardo Moscoso (Loja), Rodrigo Villegas Domínguez (Imbabura), Virgilio Mendoza (El Oro) o Julio Estupiñán Tello (Esmeraldas), en los últimos años ha alcanzado un evidente progreso en su nivel científico, mediante el concurso de un renovado corpus teórico y la inclusión de nuevas metodologías de análisis y nuevas áreas de estudio (la economía, la demografía). Empero, hoy como ayer, se desarrolla fundamentalmente gracias al esfuerzo de algunos historiadores que trabajan aisladamente en la especialidad: Willington Paredes, Jorge Trujillo, Segundo Moreno Yánez, Alfonso Anda Aguirre, Rafael Guerrero, Carmen 
Dueñas de Anhalzer, María Elena Porras, Marco Placencia, Félix Paladines, Trostky Guerrero, Luis A. León, Fernando Jurado Noboa, Genaro Eguiguren Valdivieso, Carlos Benavides Vega, Herman Flores y otros. A ellos se agregan algunos cientistas sociales que, desde una perspectiva más que nada sociológica, han incursionado en el tema de lo regional: Simón Pachano, Rafael Quintero, Erika Silva, Bertha García y Amparo Menéndez Carrión.

En el período han surgido algunos centros de investigaciones regionales, que regularmente incluyen entre su temática de estudio los asuntos históricos. Empero, el único centro de historia regional constituido en estos años fue el Archivo Histórico del Guayas, del que se habla más adelante.

La Historia de la Ciencia y la Tecnología.-Aunque poco extendida en el país, tiene algunos entusiastas cultivadores, que continúan con éxito la tradición intelectual legada por Gualberto Arcos, Misael Acosta Solís, Virgilio Paredes Borja, Mauro Madero Moreira y Enrique Garcés. Los más notables de entre los nuevos historiadores de la ciencia son Plutarco Naranjo, Rodrigo Fierro y Eduardo Estrella, todos ellos profesores de la Universidad Central del Ecuador. Naranjo y Fierro son médicos y científicos de gran prestigio, que han derivado de modo natural hacia la historia de la ciencia. Estrella une a su condición de médico una formación profesional de historiador, ha sido director-fundador del Museo Ecuatoriano de Medicina y actualmente ocupa la presidencia de la Sociedad Ecuatoriana de Historia de la Ciencia y la Tecnología. Otros nombres dignos de mención en éste ámbito son los de Domingo Paredes, profesor de la Universidad Central del Ecuador, y Jenny Estrada, investigadora del Instituto de Historia Marítima.

La Historia de la Vida Cotidiana.-Esta atractiva especialidad tiene en el Ecuador un rico antecedente, en las deliciosas Crónicas del Guayaquil Antiguo, de Modesto Chávez Franco, en los sabrosos relatos de Al margen de la historia, de Cristóbal de Gangotena y Jijón, y en las inteligentes crónicas de Camilo Destruge, Gabriel Pino Roca y Manuel J. Calle.

Dada la gran acogida que este tipo de "crónica histórica" ha tenido siempre entre el pueblo ecuatoriano, y la gran difusión que ésta alcanza a través de la prensa, hay un logrado intento contemporáneo de rescate y continuidad de ella. El esfuerzo más sostenido en este sentido ha sido el del historiador guayaquileño Rodolfo Pérez Pimentel, que desde hace años ha 
publicado regularmente sus crónicas, de corte más bien tradicionalista, en la prensa porteña. ${ }^{4}$ En el mismo espíritu se orientan las crónicas que bajo el epígrafe "Del tiempo de la yapa" publica Jenny Estrada, también en la prensa porteña, desde 1989. Posteriormente se han incorporado a esa labor, aunque con un espíritu revisionista y en busca de dar una visión alternativa a la tradicional "crónica del poder", Jorge Núñez, con sus "Historias", y Pedro Saad Herrería, con su "Calendario Histórico". 6

Por otra parte, algunos historiadores de la última generación han emprendido breves ensayos formales sobre historia de la vida cotidiana, con similar proyección. En esa línea se inscriben algunos estudios de Jorge Moreno Egas sobre las cofradías religiosas y el origen étnico de los feligreses católicos. Igualmente los de Silvia Benítez y Gaby Costa, Ximena Sosa, Cecilia Durán y María Antonieta Vásquez, cuyos trabajos figuran en la Nueva Historia del Ecuador.

La Historia Institucional.-Se impone una mención, así sea breve, a la historia institucional, subespecialidad que ha tenido un período de auge en los años reseñados. En 1973 se fundó el Instituto de Historia Eclesiástica Ecuatoriana, siguiendo el modelo de otras organizaciones afines creadas en América Latina; su finalidad es la investigación de las manifestaciones de la Iglesia Católica en la vida e historia del país y mantiene la publicación regular de un Anuario.

Pese a las limitaciones económicas que afrontan desde hace algunos años, algunas Municipalidades ecuatorianas se han esforzado en continuar, durante el período, con la publicación de sus "Libros de Cabildos", importantísimo aporte al conocimiento de las fuentes de la historia. Así, la Municipalidad de Guayaquil publicó 5 tomos, en coedición con el Archivo Histórico del Guayas; la Municipalidad de Quito, 2 tomos, y, la Municipalidad de Cuenca 3 tomos, con auspicio de la empresa Xerox del Ecuador.

En cuanto a los demás ámbitos de la historia institucional, sus resultados han sido ciertamente disímiles, por cuanto en ellos se entremezclan libros hechos en el tradicional estilo de reseña empresarial, o crónica de entidades públicas, junto con estudios propiamente históricos, que buscan

4 Estas crónicas han sido recogidas en cinco tomos, bajo el título de El Ecuador profundo.

5 Publicadas entre 1991 y 1993 en el diario Hoy, entre 1994 y 1995 en el diario El Comercio y desde fines de 1995 en el diario El Mercurio.

6 Publicado entre 1993 y 1995 en el diario Expreso, de Guayaquil. 
contribuir, desde el análisis de casos particulares, a la reconstrucción de la historia del Estado o del país. Como ejemplos del primer caso podrían mencionarse los trabajos de Enrique Boloña Rodríguez sobre la Junta de Beneficencia de Guayaquil y el comercio porteño, y del segundo, el proyecto de investigación histórica sobre el Seguro Social Ecuatoriano, desarrollado por un equipo de investigadores dirigido por Jorge Núñez, que en el breve plazo de dos años alcanzó a publicar dos tomos de fuentes (Actas de la Caja de Pensiones) y una Historia del Seguro Social Ecuatoriano.

La Historiografía Tradicional.-Pese a no ser objeto de este trabajo, creemos necesario hacer una relación al menos somera de la labor de los historiadores "tradicionales" durante el período reseñado. Si bien la obra de la mayoría de ellos ha adolecido de las limitaciones señaladas al inicio de este trabajo, no es menos cierto que lo que llamamos "vieja escuela" o "antigua generación de historiadores" no fue nunca un continente unitario sino un verdadero archipiélago, formado por islas de desigual tamaño y distinta altura. Así, entre el piélago de historiadores "tradicionales", se distinguían claramente algunos por su mayor nivel intelectual, acuciosidad investigadora o profesionalidad. Podemos citar entre estos a los siguientes: Don Alfredo Pareja Diezcanseco, un intelectual autodidacta que a sus méritos de profundo historiador - en la línea de la escuela historiográfica liberal- unía los de notable literato, lo cual le permitió escribir los textos de historia más leídos en el Ecuador del siglo XX; el padre José María Vargas, un gran investigador que incursionó por los más diversos campos del quehacer historiográfico, desde la historia del arte hasta la historia económica; Gabriel Cevallos García, historiador erudito y agudo pensador, vinculado a la escuela conservadora, en el que se funden las amplias perspectivas del "filósofo de la historia" con las intuiciones precisas del investigador; Julio Tobar Donoso, gran historiador y afamado político de derechas, conocido menos por su valiosa obra intelectual que por su desgraciada intervención en la suscripción del írrito "Protocolo de Río de Janeiro", por el que el Ecuador fue despojado de sus territorios amazónicos; Julio Estrada Icaza, historiador talentoso y regionalista intransigente, que promovió y creo con sus propios medios el afamado Archivo Histórico del Guayas; Abel Romeo Castillo, nuestro primer historiador graduado y uno de los más destacados cultores de la historia regional; Oswaldo Albornoz Peralta, pionero en la búsqueda de la "otra historia", la de los marginados y explotados del pasado; Jorge Salvador Lara, Director de la Academia Nacional de Historia y 
actual Historiador de la Ciudad de Quito, que acaba de publicar su Breve Historia Contemporánea del Ecuador; y Gerardo Nicola, pensador de orientación socialista y adelantado en la revisión crítica de los viejos métodos y teorías de la historia, lamentablemente limitado por el mundo provinciano en que le ha tocado vivir.

Desde diversos frentes de acción intelectual, todos los mencionados hicieron de puente historiográfico entre la anterior y la actual generación de historiadores ecuatorianos. De otro lado, algunos de estos acompañaron a la nueva generación durante un buen trecho del período estudiado y alguno sigue todavía en plenitud creativa. Pareja Diezcanseco fue profesor de la Escuela de Ciencias Internacionales de la Universidad Central, de la FLACSO y de la Academia Diplomática, desde donde contribuyó al desarrollo y consolidación de una moderna concepción de la historia nacional, que, sin renunciar a la defensa de los derechos amazónicos del país, apoyara el actual esfuerzo integracionista de los pueblos y países andinos. Tobar Donoso y el padre Vargas fueron profesores de la PUCE, donde contribuyeron a la creación de la Escuela de Historia y dejaron su impronta intelectual. Cevallos García fue docente de la Universidad de Cuenca y había iniciado la creación de una escuela de renovado pensamiento histórico en su ciudad natal - conocida por su gran tradición intelectual - cuando pasó a laborar en la Universidad de Puerto Rico, donde continúa hasta hoy. Estrada y Castillo renovaron en Guayaquil el interés por los estudios históricos y, a través del Archivo Histórico del Guayas, aportaron notablemente al desarrollo de las ciencias históricas en el país. En cuanto a Albornoz Peralta, en quien se juntan la herencia intelectual del radicalismo liberal y sus propias convicciones marxistas, es un historiador que ha laborado al margen del mundo académico, pero cuya influencia es notoria en un sector de nuevos historiadores ecuatorianos. Salvador Lara ha sido uno de los promotores y directivos de la Escuela de Historia de la Universidad Católica del Ecuador. Menos conocido que los anteriores, Nicola ha proyectado su pensamiento desde las instituciones educativas y culturales de Ambato, donde ha ocupado un lugar de honor.

\section{La institucionalidad existente}

En estrecha vinculación con la renovación historiográfica ecuatoriana se halla, sin duda, la presencia de una nueva institucionalidad, en general inexistente hasta antes de los años setenta. Se trata de una institucionalidad 
de variado carácter, que en ocasiones ha sido causa y en otras efecto de la transformación científico-académica producida en las últimas dos décadas. Para un análisis organizado, las hemos dividido en instituciones académicas, de promoción científica y de investigación.

Las Instituciones Académicas.-En el Ecuador contemporáneo existen dos instituciones académicas cuya labor en el campo de la enseñanza de la historia ha sido fundamental para la formación de una nueva generación de historiadores profesionales: la Pontificia Universidad Católica del Ecuador (PUCE) y la Facultad Latinoamericana de Ciencias Sociales (FLACSO), sede de Quito. A través de ello, han estimulado una renovada producción historiográfica, que ha ido multiplicándose en la última década y alimentando, en buena medida, las líneas editoriales y publicaciones periódicas especializadas.

La PUCE, a través de su Departamento de Historia, ha sido durante algunos años la institución académica de labor más sostenida, gracias al concurso de un buen equipo de docentes ecuatorianos y extranjeros. Como hemos señalado en la primera parte de este trabajo, la presencia de algunos importantes intelectuales del cono sur, que llegaran al Ecuador en calidad de refugiados políticos, coadyuvó a que esta universidad desarrollase, entre los setenta y la primera mitad de los ochenta, tanto una buena carrera de Historia cuanto toda una corriente de pensamiento histórico-filosófico, de clara raigambre americanista, que se condensó en la creación del Centro de Estudios Latinoamericanos. Gran número de alumnos acudieron a este centro de estudios y cursaron la carrera de Historia, que inclusive llegó a instituir un ciclo doctoral, dirigido por Jorge Salvador Lara.

Sin embargo, la temprana muerte del rector Hernán Malo — gran suscitador del ánimus de apertura ideológica de la PUCE—, así como los vientos de conservadurismo que empezaron a soplar sobre la iglesia latinoamericana, terminaron por ir recortando progresivamente ese espacio de amplia reflexión intelectual. En la actualidad, la especialidad se halla en franca decadencia, hecho a todas luces lamentable.

En cuanto a la FLACSO, su primer proyecto académico en Historia se produjo a partir de 1984, cuando, como parte de un proceso de reorientación interna, se abrió la Maestría en Historia Andina, teniendo como coordinador docente al historiador ecuatoriano Enrique Ayala Mora. Los objetivos del nuevo postgrado fueron: la formación de historiadores profesionales, mediante su capacitación teórica, metodológica y técnica; la 
profesionalización de la investigación histórica; el intercambio de recursos docentes en las áreas andina y latinoamericana; y el enriquecimiento historiográfico. Este esfuerzo académico fue complementado con la apertura paralela de cursos abiertos, diseñados para capacitar a alumnos no regulares de la institución. El postgrado culminó en marzo de 1986 y permitió la formación profesional de 25 alumnos, 11 de ellos ecuatorianos. Posteriormente, dificultades políticas internas provocaron el alejamiento de Ayala, con lo cual el programa de maestría quedó en suspenso. Actualmente está terminando la tercera Maestría en Historia, desarrollada bajo la coordinación del historiador peruano Heraclio Bonilla.

En síntesis, hasta la actualidad la FLACSO-Quito ha formado a dos promociones de historiadores profesionales, en parte ecuatorianos, y se halla formando a una tercera. Esto ha contribuido a profesionalizar crecientemente la investigación histórica y a enriquecer - al menos cuantitativamente- la bibliografía, gracias a la publicación de las tesis de los graduandos.

En los últimos tiempos empieza a ampliarse este panorama académico, con la creación de un Postgrado de Historia en la Facultad de Filosofía de la Universidad Central del Ecuador y de una Escuela y un Postgrado de Historia en la Universidad Estatal de Bolívar. Ello significa un impulso a la formación académica de los historiadores y augura un creciente desarrollo de los estudios históricos en el Ecuador.

Las Instituciones de Promoción Científica.--Junto a la aparición de las nuevas tendencias historiográficas en el país, surgió en Guayaquil un núcleo promotor de los estudios de historia regional, que buscó actualizar la antigua tradición historiográfica creada por el Centro de Investigaciones Históricas de Guayaquil, liderado en su hora por el ilustre historiador Pedro Robles Chambers. Este grupo intelectual alcanzó su expresión institucional en el Archivo Histórico del Guayas (AHG), cuyos principales impulsores fueran Julio Estrada Icaza y Abel Romeo Castillo. Mantenido financieramente por un Patronato privado, el AHG fue, desde entonces, quizá la más activa institución de promoción de los estudios históricos en el Ecuador. Gracias a su gestión se centralizaron todos los archivos públicos regionales: Archivo Municipal de Guayaquil, Archivo de la Casa de la Cultura Ecuatoriana, Núcleo del Guayas, y Archivo del Banco Central del Ecuador, sucursal mayor de Guayaquil. Desde 1972 mantiene su prestigiosa revista, que ha dado cabida a múltiples estudios sobre la historia local y regional de 
la costa. La labor del AHG ha sido también pionera en la tarea de vincular al país a los ecuatorianistas extranjeros y difundir localmente sus estudios, que, de otro modo, habrían permanecido ignorados por la mayoría de historiadores ecuatorianos. A mediados de 1980, el AHG pasó -mediante un convenio - a formar parte del Banco Central del Ecuador, entidad que asumió el financiamiento de sus labores y recibió en comodato las propiedades del Archivo. Lamentablemente, la enfermedad y posterior muerte de Julio Estrada Icaza, su gran motivador, y la posterior política interna del $\mathrm{BCE}$, encaminada a deshacerse de todas las tareas socio-culturales que desarrollaba anteriormente, han determinado que el AHG se halle desde 1990 en un estado de virtual abandono y que su valiosa revista haya sido descontinuada.

De otro lado, hemos analizado al comienzo de este trabajo el proceso constitutivo del movimiento de renovación historiográfica ecuatoriana y en ello se evidencia el importante papel cumplido en el Ecuador por la Asociación de Historiadores Latinoamericanos y del Caribe (ADHILAC), que ha mantenido sucesivamente en el país la sede de su Secretaría Ejecutiva (1981-1990) y de su Consejo Directivo Internacional (1990-1994). En este sentido, el mayor logro alcanzado ha sido la constitución de la Asociación de Historiadores Ecuatorianos (ADHIEC), en 1986, y la organización de la Asociación Nacional de Profesores de Historia (ANPHI), en 1991.

A través de la organización de los Encuentros de Historia Nacional, el reciclaje científico de los profesores universitarios y secundarios, la estimulación de proyectos investigativos y la publicación de libros, la ADHIEC ha cumplido hasta el momento una buena labor de promoción científica. En cuanto a la ANPHI, dada su corta vida es todavía una promesa de futuro.

En cuanto se refiere a la Academia Nacional de Historia, fundada a comienzos de siglo por el arzobispo-historiador Federico González Suárez, con el nombre de Sociedad de Estudios Históricos Americanos, su vinculación al más conspicuo tradicionalismo social e ideológico la ha mantenido en las últimas décadas voluntariamente al margen de las nuevas corrientes historiográficas, frente a las que ha sostenido una actitud más bien pasiva y distante. Estragada por el tiempo y por su propia falta de renovación interna, la Academia ha dejado de ser el alto cenáculo intelectual que fuera otrora y ha terminado por convertirse en un reducto de gentes de derechas, algunas sin nivel académico y, en ciertos casos, inclusive sin ninguna 
obra intelectual. Pese a todo ello, mantiene todavía cierta ocasional actividad y ha organizado algún evento científico de importancia durante la pasada década, aunque sin generar una línea de pensamiento histórico ni una corriente de acción historiográfica. Su única labor significativa, y ciertamente encomiable, es la publicación de su Boletín científico, que se mantiene ininterrumpidamente desde la fundación de la entidad, en 1909. Carente de una actividad institucional que lo sustente, el Boletín sale hoy gracias al entusiasmo personal de Jorge Salvador Lara y pese a las limitaciones económicas de esta institución, que no cuenta con un adecuado respaldo financiero por parte del Estado.

Los Organismos y Medios de Difusión.-No hay historiografía posible sin el concurso de las instituciones de difusión, que a través de sus publicaciones ponen en contacto al investigador y al lector. Labor relevante ha sido, a este propósito, la de algunas editoriales ecuatorianas, que han respaldado la labor de los nuevos historiadores y promocionado sus estudios, llegando en algunos casos a crear colecciones o bibliotecas editoriales especializadas en historia.

Entre estas debemos destacar a la Corporación Editora Nacional (CEN), que ha desarrollado una labor excepcional y cuenta entre sus variados logros la publicación de la Biblioteca Básica del Pensamiento Ecuatoriano (40 tomos, en coedición con el BCE), del Libro del Sesquicentenario (4 tomos), de la Nueva Historia del Ecuador -ambiciosa obra generacional en 15 tomos, de la que ya hemos hablado anteriormente - y de la revista de historia Procesos, de joven vida y prometedor futuro. Avanza también la publicación de la Biblioteca de Historia Ecuatoriana (11 tomos), la Biblioteca ecuatoriana de Arqueología (5 tomos), la Colección Testimonios (11 tomos), la Biblioteca de Ciencias Sociales (41 tomos) y otras colecciones de contenido historiográfico.

También ha sido importante en este sentido la labor de la Editorial "El Conejo", que publica periódicamente estudios de historia republicana, promueve la preparación de estudios sobre historia contemporánea, difunde su propia biblioteca editorial de historia ecuatoriana y edita las revistas culturales Palabra Suelta (Quito) y Matapalo (Guayaquil), siempre abiertas a la publicación de ensayos históricos.

Otra institución que ha trabajado con entusiasmo por la difusión histórica es el ya fenecido grupo CEDIS-CEDEP, que publicara varios estudios históricos y cuyos herederos, los ahora independientes CEDIS y CEDEP, 
han mantenido una similar línea de ediciones; en el caso del CEDEP, ello se ha ampliado a la edición de vídeos y radionovelas de inspiración histórica, siempre destinados a la educación popular.

Empero, la labor editorial más impresionante ha sido la de la editorial salesiana "Abya Yala" ("Tierra Nuestra" en idioma shuar), que en 1992 cumplió sus primeros diez años de vida con la publicación de su libro número 500, con el mérito adicional de que la mayoría de obras publicadas contienen nuevas investigaciones. Con escasos recursos pero animada por el enorme entusiasmo de Juan Botasso y Javier Juncosa, esta editorial ha puesto al alcance del público ecuatoriano y latinoamericano una gran variedad de estudios históricos y antropológicos, en ediciones de digna presentación y bajo costo. Al momento su producción editorial alcanza ya el millar de títulos.

Similar en magnitud e importancia ha sido la labor emprendida por la Universidad de Guayaquil con la publicación de su Biblioteca Ecuatoriana, que en buena medida ha contemplado la publicación de nuevos estudios históricos y la reedición de otros que, por su rareza, casi eran imposibles de conseguir. Detrás de ello ha estado la labor de un notable y acucioso historiador, Elías Muñoz Vicuña, quien ha seleccionado los textos y hecho los estudios introductorios de la colección.

Por fin, es necesaria una mención particular a las revistas y boletines científicos especializados. Además de los ya mencionados boletines y revistas de historia (Revista del Archivo Histórico del Guayas, del BCE; Miscelánea Histórica Ecuatoriana, del BCE; Cuadernos de Historia y Arqueología, de la Universidad de Guayaquil; Boletín de la Academia Nacional de Historia; Boletín del Instituto de Historia Eclesiástica Ecuatoriana; Revista Ecuatoriana de Historia de las Ideas, coeditada por la PUCE y la CCE; Procesos, de la CEN; Memoria, de MARKA, Museo Histórico, de la Municipalidad de Quito), existen en el país las siguientes publicaciones ocasionales de historia: ARNAHIS, revista del Archivo Nacional de Historia; la Revista de Historia y Geografía de la CCE; la Revista del Instituto de Historia Marítima, y, la Revista del Centro Nacional de Investigaciones Genealógicas y Antropológicas.

Adicionalmente, fuera de la ya mencionada revista Cultura del BCE, existen algunas otras revistas culturales o de ciencias sociales que de modo regular publican artículos históricos: Anales de la Universidad Central (la más antigua publicación científica ecuatoriana); Revista de la Universidad de Guayaquil; Miscelánea Antropológica Ecuatoriana, del BCE; Ecuador 
Debate, revista de ciencias sociales del CAAP; Caspicara, revista cultural de la Dirección de Centros Culturales del Municipio de Quito; Nariz del Diablo, revista de ciencias sociales del CEDIS, y, Revista de la Fuerzas Armadas Ecuatorianas.

Como una agradable nota final a este capítulo hay que referirse a dos importantes esfuerzos historiográficos privados: la Colección de Historia del Grupo automotriz AYMESA, editada desde 1992 por la Fundación del mismo nombre y que hasta el momento lleva publicados diez tomos; y la colección de libros de historia del arte ecuatoriano publicada por el hoy desaparecido Banco de los Andes, en ediciones de gran calidad intelectual y gráfica.

\section{La historiografía ecuatorianista}

No estaría completo el panorama de la actual historiografía ecuatoriana sin una mención, así sea breve, de los aportes hechos a ella por los ecuatorianistas extranjeros, quienes, sin otra motivación que el conocimiento científico en si mismo, se han dedicado al estudio de la historia ecuatoriana. A tal propósito, este trabajo no pretende sino aportar unas breves apreciaciones generales, que den idea de la magnitud y perspectiva del aporte de los ecuatorianistas; el análisis adecuado de esta contribución requiere necesariamente de un estudio detenido, que escapa a los límites de esta ponencia.

Una primera y necesaria observación que debe plantearse es la referida a la calidad y variedad de esos aportes. En cuanto a su calidad, podemos afirmar que en general se trata de trabajos de buen nivel, elaborados a base de una exhaustiva búsqueda de fuentes primarias y un minucioso procesamiento de datos. En lo que dice de su variedad, nos hallamos frente a una sorprendente diversidad de temáticas tratadas por los historiadores ecuatorianistas, la que inclusive abarca temas poco o nada estudiados por los historiadores ecuatorianos.

Complementando esta primera observación, creemos necesario evaluar el impacto que los aportes de los ecuatorianistas extranjeros han causado en la propia historiografía ecuatoriana. Por la misma minuciosidad y profesionalismo con que han sido preparados, esos trabajos producen un positivo efecto entre nosotros, tanto porque contribuyen a enriquecer la masa de conocimientos comprobados que poseemos sobre nuestro pasado 
como porque ofrecen nuevas perspectivas y metodologías de análisis y, últimamente, porque suscitan una saludable emulación entre los científicos sociales ecuatorianos.

Pero no todo es color de rosa en el campo de la historiografía ecuatorianista: el aporte metodológico de la mayoría de los historiadores extranjeros tiene también unas limitaciones objetivas y, en cierta perspectiva, inclusive unos efectos nocivos. Así, se puede apreciar que se ha producido en el período una gran acumulación positivista de monografías y artículos menores, que muchas veces no tienen más sustento informativo que algún documento suelto encontrado al azar. Ello, a su vez, ha generado una corriente local de "monografismo", que generalmente se mueve por las pautas y modas temáticas llegadas del extranjero. Vista en bloque, esa acumulación monográfica - tanto ecuatoriana como ecuatorianista- ha permitido recoger una cantidad muy grande de información sobre nuestro pasado, pero, por su mismo enfoque reduccionista, nos ha ayudado sólo limitadamente a comprender mejor nuestra historia: su amplio horizonte social, su largo plazo, sus grandes fenómenos.

Limitaciones aparte, es innegable que muchos estudios de los historiadores ecuatorianistas son de gran calidad y perspectiva verdaderamente científica, y que inclusive han cubierto los vacíos dejados por los historiadores ecuatorianos a causa del mismo escaso y tardío desarrollo de nuestras ciencias históricas; en otros casos, es notorio que esos aportes han enriquecido significativamente la labor ya emprendida por los historiadores nacionales, especialmente en lo que tiene que ver con la historia regional.

Quiero citar como ejemplo de lo dicho el caso de la historia regional de Guayaquil, en el cual los aportes de los ecuatorianistas han sido francamente notables, destacándose entre ellos los estudios del norteamericano Michael Hamerly, en especial su difundida Historia social y económica de la antigua Provincia de Guayaquil. 1763-1842, del también norteamericano Adam Szászdi y su esposa Dora León Borja, del británico David J. Cubitt y sobre todo de la española María Luisa Laviana Cuetos, cuyo estupendo libro Guayaquil en el siglo XVIII. Recursos naturales y desarrollo económico es, con seguridad, el mejor y más completo estudio que existe sobre una región ecuatoriana en ese período y, a su vez, el más importante hito en la ya larga saga de estudios hechos por su autora respecto de la historia guayaquileña.

Buena muestra de la labor "ecuatorianista" en el campo de los estudios de historia regional ha sido también el "Proyecto Loja", desarrollado 
entre 1980 y 1983 en la región sur del Ecuador por el Instituto Francés de Estudios Andinos (IFEA), en colaboración con el Banco Central del Ecuador, y cuyo sumario de informes fuera recogido por la Revista Cultura del Banco Central del Ecuador en su número 15. Este proyecto tuvo un alcance trascendental para la región estudiada, pues muchos de los temas que abarcó simplemente no habían sido tratados hasta entonces por los historiadores u otros científicos ecuatorianos. Dentro de un amplio equipo binacional de investigación científica, colaboraron en este proyecto algunos historiadores y arqueólogos franceses, como Chantal Caillavet, Martín Minchom, Ives Saint-Geours, Martine Petitjean, Emmanuel Fauroux, Jean Guffroy, Patrice Lecoq y Antonio Fresco.

En la nómina de prestigiosos científicos extranjeros que han desarrollado sucesivos estudios sobre la historia ecuatoriana ocupan también lugar de honor el español Javier Ortiz de la Tabla, autor de muchos ensayos importantes sobre la historia social, económica y demográfica de la región andina ecuatorial, culminados con su excelente obra Los encomenderos de Quito. 1534-1660; el norteamericano Frank Salomon, que ha aportado a nuestra historiografía una obra intelectual de gran calidad y variedad, y los alemanes Udo Oberem, notable sabio y antiguo estudioso de los temas ecuatorianos, y Christiana Borchart de Moreno, prestigiosa investigadora de los temas de historia social y económica. Dentro de este grupo de "ecuatorianistas de tiempo completo" debemos incluir también a la ya citada historiadora francesa Chantal Caillavet, cuya obra intelectual sobre el Ecuador es ciertamente relevante.

De modo menos frecuente, pero con una alta calidad investigativa, se han ocupado asimismo de temas de la historia ecuatoriana el francés Bernard Lavallé, el inglés Malcolm Deas, los españoles Demetrio Ramos Pérez, José Alcina Franch, Luis J. Ramos Gómez, Manuel Lucena Salmoral, Antonio Lafuente, Leoncio López Ocón y Berta Ares Queija, los norteamericanos John Murra, Magnus Mörner, Nick D. Mills Jr., Linda Alexander Rodríguez, Robson Brines Tyrer, Eric Beerman y Lois Crawford de Roberts, y el chileno Horacio Larraín, entre otros. Sus aportes al conocimiento histórico del pasado ecuatoriano, así como sus interpretaciones teóricas y planteamientos metodológicos, han sido ciertamente de gran utilidad para el desarrollo de las ciencias históricas en el Ecuador.

Un tercer grupo de ecuatorianistas lo constituyen aquellos que han trabajado ocasionalmente algún tema de nuestra historia y cuya producción se reduce a una sola publicación. Figuran entre ellos las francesas Anne 
Christine Taylor e Iveline Lebret, el español Antonio Mazuecos y los norteamericanos John L. Phelan, Nicolas Cushner, Allan J. Kuethe, John C. Super y Paul Drake, y Ricardo Muratorio. Este es también, en general, el caso de los estudiantes extranjeros de la FLACSO, que han aportado y aportan a nuestra historiografía con sus trabajos de tesis, y también el de ciertos asistentes técnicos extranjeros, que efectúan investigaciones históricas durante su permanencia en el país y de modo preferente sobre temas de la región o localidad en donde desempeñan su labor profesional. Entre estos egresados flacsonianos destacamos nombres como los de Carlos Contreras, Leoncio López Ocón o Diana Bonnett, los primeros de los cuales ha seguido cultivando luego su interés por la historia ecuatoriana y efectuando aportes ocasionales a nuestra historiografía.

Desde luego, esa falta de continuidad en el interés de los "ecuatorianistas iniciales" está causada, en buena medida, por la despreocupación con que las instituciones oficiales ecuatorianas han manejado casi siempre el asunto, desatendiendo la labor de estos amigos del país y no ofreciendo casi ningún estímulo a su generoso trabajo intelectual. La única excepción a esta actitud se dio, probablemente, durante el gobierno del presidente Rodrigo Borja, en el cual, desde la Subsecretaría de Cultura, invitamos a los ecuatorianistas españoles a participar con sus colegas del Ecuador en una primera reunión de intercambio científico alrededor del tema "Fuentes para la historia ecuatoriana". Pese a su notable éxito inicial y a las expectativas que abrió, este esfuerzo no tuvo continuidad, pues no se efectuó una segunda reunión de ese tipo que debía realizarse en España, bajo convocatoria de los historiadores españoles; empero, permitió un contacto directo entre historiadores de ambos países, mismo que posteriormente ha fructificado en diversas formas de cooperación. En esta misma línea, la Subsecretaría de Cultura otorgó la "Condecoración Nacional al Mérito Cultural" a dos historiadores españoles que figuran entre los ecuatorianistas más notables, María Luisa Laviana Cuetos y Javier Ortiz de la Tabla Ducasse, quienes han reciprocado luego estas muestras de estímulo oficial con una renovada preocupación por los asuntos de la historia ecuatoriana.

Muy inteligente y objetiva ha sido, en este campo, la actitud de algunas entidades culturales privadas o autónomas, que se han interesado constantemente por el trabajo de investigación de los ecuatorianistas extranjeros, invitándolos periódicamente a participar en simposios científicos o publicando sus trabajos. Respecto de esa labor, son particularmente recomendables las acciones institucionales de la ADHILAC-ADHIEC, de la 
FLACSO y de la Universidad Andina "Simón Bolívar", que constantemente organizan reuniones científicas con historiadores ecuatorianistas, así como las del Archivo Histórico del Guayas, de la Corporación Editora Nacional y de la revista Cultura del Banco Central del Ecuador, que han instituido la publicación regular de libros o artículos de historiadores extranjeros.

En beneficio de la misma historiografía ecuatoriana y de sus cultores sería de desear que el Estado y las instituciones culturales públicas pusieran mayor interés en el trabajo de los historiadores ecuatorianistas, quienes sirven desinteresadamente a los intereses del país y regularmente no reciben un adecuado estímulo de nuestra parte.

Pueden plantearse algunas tareas útiles a este propósito: levantar un banco de datos sobre su labor, con información actualizada; interesarse en publicar sus trabajos y mantener con ellos una relación permanente, por medio de las instituciones culturales nacionales y nuestras representaciones diplomáticas en el extranjero; promover proyectos de investigación en los que cooperen historiadores ecuatorianos y extranjeros, etc. Por lo demás, un reiterado contacto entre los historiadores ecuatorianos y los ecuatorianistas extranjeros será siempre de gran utilidad científica, en razón de las diversas y complementarias fuentes que manejan unos y otros, y del enriquecedor cotejo de criterios y experiencias que puede darse.

Como dijéramos antes, parte sustantiva del aporte de los historiadores ecuatorianistas ha radicado en su preocupación por especialidades poco cultivadas por los historiadores ecuatorianos, como, por ejemplo, la historia demográfica y la historia económica. En efecto, han sido los estudiosos extranjeros quienes iniciaron los trabajos de historia demográfica sobre el Ecuador y también quienes han realizado los mayores aportes en la especialidad, debiendo ponerse de relieve los trabajos de Michael T. Hamerly, Rosemary D. F. Bromley, Javier Ortiz de la Tabla, Robson Brines Tyrer, Martin Minchom y Karen Powers Vera.

En el campo de la historia económica sucede algo similar, pues la especialidad se ha desarrollado básicamente por los aportes de los ecuatorianistas extranjeros. Mientras Brines Tyrer se empeñaba en un estudio general de la economía colonial del país, la mayoría de investigadores extranjeros optaban por centrar su enfoque en áreas regionales, debiendo subrayarse en esta perspectiva los trabajos del mismo Hamerly, de Laviana Cuetos, de los esposos Szászdi, de Cubbit y de Crawford sobre el área de la costa central, así como los estudios del notable investigador colombiano 
Germán Colmenares, prematuramente fallecido, sobre las haciendas jesuitas de la sierra norte y los métodos de acumulación originaria de la Compañía de Jesús. Igualmente, son de gran importancia los estudios de Ortiz de la Tabla y Borchart sobre la economía colonial en la sierra centro-norte, de Caillavet y Saint-Geours sobre la región lojana, de Palomeque sobre la región azuaya y de Alexander sobre las reformas del movimiento "juliano", vistas como un enfrentamiento político-económico regional.

Hemos señalado también en líneas anteriores el papel protagónico que los ecuatorianistas han tenido en el desarrollo de la historia regional, ya complementando los estudios locales - como ocurrió en Guayaquil y el Azuay - o ya motivándolos, como en el caso de Loja.

Llegados a este punto, se impone una reflexión sobre las inclinaciones temáticas de la generalidad de historiadores ecuatorianistas. Es evidente, por ejemplo, su preferencia por los temas de historia económica y demográfica, la arqueología o la etnohistoria, que se expresa tanto en los estudios generales como en los regionales. Ello obedece ciertamente a las condiciones objetivas que existen en sus países para la investigación histórica, tales como la existencia de un financiamiento preferencial para temas vinculados a ciertas áreas de "su" interés nacional (lo económico), o la presencia de "temáticas de moda" (ayer lo demográfico, hoy lo ecológico), detrás de las cuales incluso es relativamente fácil identificar a los principales "modistos" de cada país (p. e., en los EE. UU, el grupo de historia demográfica de Berkeley: W. Borah, S. F. Cook y L. B. Simpson).

Mas, por otra parte, también salta a la vista su desinterés por los temas de la historia política, en general, y de la específicamente republicana, en particular. ¿Cuáles podrían ser las motivaciones de ese desinterés? En nuestra opinión, en la base de esa actitud hay, además de las inclinaciones de la moda intelectual, una inconsciente mezcla de juicios y prejuicios culturales (religiosos, étnicos, políticos) prevalecientes en Europa y los Estados Unidos respecto de las repúblicas latinoamericanas, y que tienen que ver con el carácter mestizo de su población, con su tradicional inestabilidad política y con su misma vocación republicana.

Tomemos por ejemplo a los ecuatorianistas norteamericanos. Muchos de ellos - especialmente los etnohistoriadores - actúan con un espíritu abierto y desprejuiciado y muestran un notable interés por nuestra realidad pasada y presente. Empero, existe también un gran número de ellos que se aproxima a nuestra historia sobre los rieles de unos conceptos preconcebidos. Dicho de otro modo, no buscan estudiarla a partir de si misma, en su 
particular marco socio-cultural, sino a partir de los parámetros de su propia cultura. Así, ante sus ojos de buenos calvinistas o protestantes, el catolicismo de los pueblos del sur, lleno de manifestaciones externas (ricos templos, imágenes polícromas, procesiones masivas), aparece como un culto fanático e idolátrico, y legitima su autovaloración de "pueblo escogido".

En otros casos, el análisis se efectúa a partir de los particulares conceptos norteamericanos de "democracia", "ley" y "orden". Cabal ejemplo de ello es el estudio de Adam Szászdi sobre la historiografía republicana del Ecuador, texto elaborado con bastante profesionalidad pero animado por una ideología absolutamente reaccionaria, donde se entremezclan el anticomunismo, el antiliberalismo y el filoconservadurismo más apasionados. Uno de los fenómenos más trascendentales de la historia republicana, cual es la Revolución Liberal de 1895, y su líder máximo, Eloy Alfaro, son juzgados en este trabajo con un espíritu totalmente prejuiciado, a la par que se exalta la figura del gran dictador, Gabriel García Moreno, hasta el extremo de justificar sus peticiones de protectorado francés para el Ecuador y su respaldo a la intervención francesa en México.

En el caso de los historiadores europeos, salta a la vista que la mayoría de sus trabajos sobre la historia del Ecuador - y, en general, sobre la de América Latina - enfocan temas referidos a la época colonial. Ello es explicable en tanto que tienen en sus propios archivos las fuentes referidas al período colonial, mientras que les quedan lejanas las fuentes del período republicano. Pero no es menos cierto que, junto a esas distancias físicas, cada vez más disminuidas por la tecnología moderna, se percibe un "distanciamiento mental", quizá inconsciente, de los latinoamericanistas europeos con relación a la historia republicana de América Latina, al punto que ello justificaría especular con la idea de que, en general, les interesa nuestra historia en cuanto puede ser vista como apéndice de la suya propia.

\section{Palabras finales}

Al terminar este trabajo creemos necesario resumir en unas pocas líneas la evaluación historiográfica del último cuarto de siglo.

La sola cantidad de información que hemos debido recoger para emprender el presente ensayo, pese a tratarse de una información inevitablemente incompleta, revela ya que estamos ante un gran salto cuantitativo de la historiografía ecuatoriana. Nunca antes se había producido tanto 
durante un período similar. Nunca antes se había incursionado en tantos temas o se habían formulado tan variadas interpretaciones teóricas. Nunca antes había existido tan gran número de gentes dedicadas a la investigación histórica. Nunca se había publicado tanto.

También el salto cualitativo ha sido notable, aunque lamentablemente inferior al cuantitativo. Sin embargo, es irrefutable que ese salto de calidad se ha producido en el último cuarto de siglo y que sus manifestaciones más evidentes son las siguientes:

a) La generalizada superación del "culto a los héroes" como vocación y de la biografía como género, y su sustitución por una visión crecientemente científica de la historia, expresada en numerosos estudios acerca de la estructura socio-económica y de los procesos vinculados a ella (sociales, económicos, culturales, demográficos), o en investigaciones sobre la coyuntura y sus fenómenos.

b) El abandono del anterior ejercicio historiográfico, las más de las veces limitado al "refrito" de datos ya conocidos y a la glosa de opiniones ajenas, y el surgimiento de un renovado interés por la investigación histórica, por ese "regreso a las fuentes de la historia" por el que clamaba Gabriel Cevallos García a fines de los años cincuenta. ${ }^{7}$

c) La superación del relato cronologista, de los grandes "nudos historiográficos" y del empirismo metodológico en beneficio de la reflexión histórica, la amplitud temática y una vocación plenamente cientificista.

d) La presencia de una "historia crítica" o revisionista en el lugar que antes ocupaba la "historia oficial", y la generalizada preocupación por investigar la historia de los sectores sociales marginados (clases, etnias), de las regiones olvidadas y de los períodos poco conocidos.

Desde luego, el desnivel existente entre la cantidad y la calidad de la actual historiografía ecuatoriana no es un asunto atribuible a la voluntad personal de los historiadores. Muchas circunstancias han determinado que esto fuera así y no de otro modo ni de mejor manera. Entre ellas se destacan algunas que no queremos soslayar y que son las siguientes:

La poca preocupación del Estado y los poderes públicos por los asuntos de la investigación histórica, lo que contrasta penosamente con la verborrea historicista de que, en general, hacen gala todos los políticos y

7 Reflexiones sobre la historia del Ecuador, 2 tomos, Cuenca, 1957, tomo 1, pág. 11. 
gobernantes ecuatorianos (No hay centros de investigación, no hay becas de investigación, no hay una política de investigación).

La lamentable situación en que se hallan los archivos públicos ecuatorianos, que, como el Archivo Nacional de Historia, carecen hasta de un local propio y viven arrimados a la sombra de otras instituciones (en este caso, de la CCE). Adicionalmente, el Sistema Nacional de Archivos sólo existe en la letra de la ley que lo creo, pues carece de recursos para recoger, catalogar y poner en uso, en todo el país, los fondos documentales cuya protección le ha sido legalmente encargada.

La prolongada crisis académica de las universidades nacionales, que, salvo alguna honrosa excepción, se hallan del todo imposibilitadas para formar historiadores o para sostener una coherente política de investigación histórica y publicaciones.

La falta casi total de condiciones para la profesionalización de los historiadores, cuya labor es vista por el público, y en primer lugar por el Estado, como una erudita tarea recreativa que no necesita ni merece remuneración alguna. (Todavía es común que las instituciones, periódicos, revistas, etc, nos inviten a dictar una conferencia o escribir un artículo sin sentirse en la obligación de pagar por ello).

Frente a escollos tan grandes y aparentemente insalvables, poco es lo que personalmente pueden hacer los historiadores, quienes ya tienen bastante con darse modos para asegurar su supervivencia en medio de la crisis económica, porque, como se sabe, el oficio de historiador no es redituable. Corresponderá, pues, a las organizaciones gremiales, y en primer lugar a la Asociación de Historiadores del Ecuador (ADHIEC), el esfuerzo por cambiar esta situación y alcanzar para los historiadores ecuatorianos unas condiciones mínimas de trabajo profesional.

En fin, es a la sombra de este panorama crítico que debe valorarse el aporte científico de los historiadores ecuatorianos contemporáneos. Sinceramente, creo que su entrega a la historiografía del país ha sido notable y que, si se remueven los escollos señalados, puede ser inmensamente mayor. Pero esa es ya una tarea de futuro. 